\title{
Erratum to: Predictors of Preterm Births and Low Birthweight in an Inner-City Hospital in Sub-Saharan Africa
}

\author{
Bolajoko O. Olusanya $\cdot$ Gabriel E. Ofovwe
}

Published online: 23 September 2010

(C) Springer Science+Business Media, LLC 2010

\section{Erratum to: Matern Child Health J DOI 10.1007/s10995-009-0528-4}

The authors wish to advice the following corrections consequent on the use of 'row' rather than 'column' percentages in the formula $\mathrm{Pe}(\mathrm{OR}-1) / \mathrm{OR}$ as stated under the statistical methods.

\begin{abstract}
Replace penultimate sentence with "Premature rupture of membranes $(\mathrm{PAR}=33.91 \%)$ and antepartum hemorrhage $(\mathrm{PAR}=33.54 \%)$ were the leading contributors to preterm birth in contrast to IUGR $(\mathrm{PAR}=82.28 \%)$ and premature rupture of membranes $(\mathrm{PAR}=32.31 \%)$ for low birthweight."
\end{abstract}

\section{Results}

Risk factors for preterm birth

Replace fifth sentence with "Premature rupture of membranes $(\mathrm{PAR}=33.91 \%)$ and antepartum hemorrhage (PAR = $33.54 \%$ ) had the highest attributable risk for preterm delivery."

Risk factors for low birthweight

Replace third sentence with "IUGR was not only associated with the largest odds but also with the highest attributable risk for low birthweight $(\mathrm{PAR}=82.28 \%)$ followed by premature rupture of membranes (PAR $=32.31 \%)$."

Table 3

Sex for Low birthweight: Male 195 (8.7\%); Female 245 $(11.9 \%)$.

\section{Table 4}

The revised PAR values in the model for Prematurity are: Single/unmarried (13.0\%); Small trading (8.56\%); Full-time job $(4.43 \%)$; Shared sanitation $(4.46 \%)$; Lack of antenatal care (4.83\%); No previous cesarean section (6.03\%); Hypertensive disorders (19.58\%); Antepartum hemorrhage (33.54\%); Premature rupture of membrane (33.91\%); Maternal disease $(9.33 \%)$.

The revised PAR values in the model for Low birthweight are: Shared sanitation (2.68\%); Lack of antenatal care (8.61\%); No previous cesarean section (4.78\%); Hypertensive disorders (12.14\%); Premature rupture of membrane (32.31\%); Gender (3.02\%); Intrauterine growth restriction (82.28\%). 